\title{
Volume dependence of light hadron masses in full lattice QCD
}

\author{
B. Orth ${ }^{\mathrm{a}}$, T. Lippert ${ }^{\mathrm{a}}$ and K. Schilling ${ }^{\mathrm{a}}$

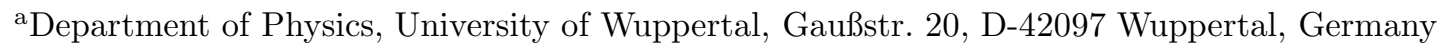

The aim of the GRAL project is to simulate full QCD with standard Wilson fermions at light quark masses on small to medium-sized lattices and to obtain infinite-volume results by extrapolation. In order to establish the functional form of the volume dependence we study systematically the finite-size effects in the light hadron spectrum. We give an update on the status of the GRAL project and show that our simulation data for the light hadron masses depend exponentially on the lattice size.

\section{INTRODUCTION}

Lattice QCD has always been plagued by the fact that towards realistic quark masses the simulation costs increase drastically due to the large correlation lengths of the light states and the large lattice volumes that one employs in order to avoid finite-size (FS) effects. This difficulty has, in fact, prevented us until this day from carrying out simulations with truly physical parameters. Instead one resorts to computations with several un-physically large quark masses in FSeffect-free volumes, followed by an extrapolation to the physical quark mass. However, the chiral extrapolation introduces uncertainties that can be reduced only by increasing the overlap of lattice simulations with ChPT. It is thus inevitable to look out for feasible ways to obtain lattice results at light quark masses. Current approaches like overlap, domain-wall or improved staggered fermions are still very costly, especially if one goes beyond the quenched approximation. Faced with limited computer resources we therefore investigate the feasibility of an approach that restricts itself to the use of standard Wilson fermions.

The aim of the GRAL project is to carry out a systematic study of FS effects in the light hadron spectrum in the parameter regime accessible to us. With simulations on series of small to medium-sized lattice volumes (at fixed coupling and quark mass, respectively) we also want to address the issue of whether one can use formulae obtained from this investigation to extrapolate hadronic observables to the infinite volume.

\section{VOLUME DEPENDENCE OF THE LIGHT HADRON MASSES}

In 1983 M. Lüscher published a universal field theoretic formula for the shift in the mass of a stable particle enclosed in a finite box [12]. The formula states for asymptotically large volumes that the FS mass-shift vanishes exponentially with increasing box size at a rate that depends on the particle considered and on the spectrum of light particles in the theory. If, in QCD, we consider a stable hadron in a box of size $L^{3}$ with periodic boundary conditions and sufficiently large (Euclidian) time-extent $T$, its mass $M_{H}(L)$ becomes a universal function of $M_{\pi} L$ (where, at fixed $\beta$, $\left.M_{\pi}=\lim _{L \rightarrow \infty} M_{\pi}(L)\right)$ in the finite-volume continuum limit. Since FS effects probe the system at large distances and are thus insensitive to the form and magnitude of the UV cutoff, this function is expected to hold also for finite lattice spacings. With the (constant) LO chiral expression for the $\pi-\pi$ forward scattering amplitude the relative mass-shift for the pion is given by

$$
\begin{aligned}
\frac{M_{\pi}(L)-M_{\pi}}{M_{\pi}} & =\frac{3}{8 \pi^{2}} \frac{M_{\pi}^{2}}{F_{\pi}^{2}} \frac{K_{1}\left(M_{\pi} L\right)}{M_{\pi} L} \\
& \sim \frac{3}{4(2 \pi)^{3 / 2}} \frac{M_{\pi}^{2}}{F_{\pi}^{2}} \frac{e^{-M_{\pi} L}}{\left(M_{\pi} L\right)^{3 / 2}}
\end{aligned}
$$

If we assume, for simplicity, the pion-nucleon forward scattering amplitude to be constant, too, then the formula for the nucleon involves a term that is - up to low-energy constantsproportional to the one for the pion. In addition 
it contains a term proportional to

$$
\exp \left(-M_{\pi} L \sqrt{1-M_{\pi}^{2} /\left(4 M_{N}^{2}\right)}\right) /\left(M_{\pi} L\right) \text {. }
$$

On the other hand, in 1992 Fukugita et al. found a power-like $L$-dependence of the form

$M_{H}(L)=M_{H}+c / L^{3}$

in their data for the pion, rho and nucleon masses 34. The usual explanation for the apparent discrepancy is that Lüscher's formula deals with asymptotically large lattice volumes where FS effects arise from a squeezing of the virtual pion cloud surrounding the hadron in a box with periodic boundary conditions. In contrast, Fukugita et al. ascribe the power-like behaviour of their data to a squeezing of the hadron itself (as it would occur for small lattice volumes). In this picture one expects a power-like dependence for sub-asymptotic lattice volumes that gradually changes into an exponential fall-off of the hadron masses towards larger box sizes.

\section{GRAL STATUS}

Table 1 summarises some details of our simulations. We use the standard unimproved Wilson action with two degenerate flavours of dynamical fermions. Our HMC codes are running on the APEmille at DESY/Zeuthen and on the cluster installation ALiCE at Wuppertal University. In addition to the already available SESAM $(L=16)$ and $\mathrm{T} \chi \mathrm{L}(L=24)$ ensembles we are generating configurations on smaller volumes with $L$ varying between 10 and 16 . The figures referring to these runs are, where available, preliminary. With our simulations at $\beta=5.32$, $\kappa=0.1665$ we aim at a quark mass corresponding to $M_{\pi} / M_{\rho} \approx 0.5$, i.e. below the smallest $M_{\pi} / M_{\rho}$ of $0.575(16)$ previously achieved by the $\mathrm{SESAM} / \mathrm{T} \chi \mathrm{L}$ project. In this article we focus on the ensembles at $\beta=5.6, \kappa=0.1575$ where we assume the largest, $24^{3} \times 40$-lattice to be free of FS effects.

\section{RESULTS}

The (preliminary) masses we obtain for the pion, rho and nucleon are displayed in Fig. 11.

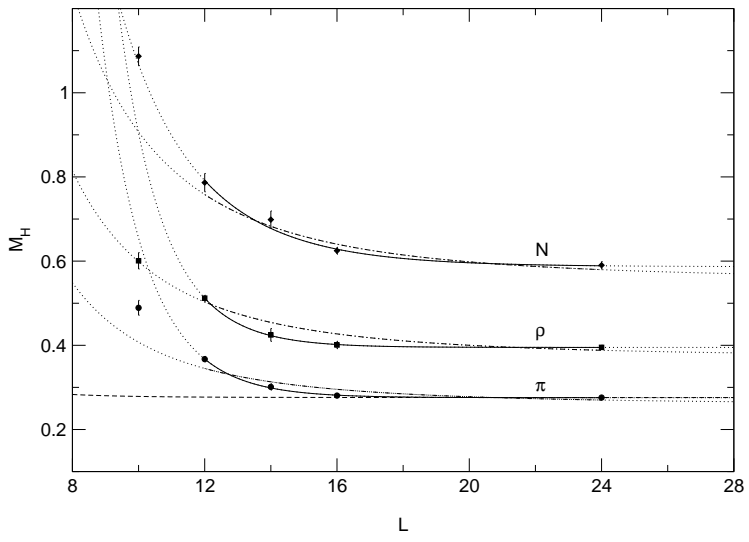

Figure 1. Dependence of the $\pi$-, $\rho$ - and $N$-masses for $\beta=5.6, \kappa=0.1575$ on the spatial lattice extent, $L$. The curves are explained in the text.

The flat dashed curve represents Lüscher's formula for the pion mass, Eq. (10), with $M_{\pi}$ and $F_{\pi}$ as obtained from the $24^{3}$-lattice. From this curve it is obvious that the data points at $L=$ $10,12,14$ are outside the chiral regime. This can also be seen from $F_{\pi} L$, which takes the values $0.91(3), 1.10(3), 1.28(4), 1.46(4)$ and $2.19(6)$ for $L=10,12,14,16,24$, respectively. Since ChPT is a low-energy (large distance) expansion it is only valid for $F_{\pi} L \gg 1$ and $M_{\pi} /\left(4 \pi F_{\pi}\right) \ll 1$; $M_{\pi} /\left(4 \pi F_{\pi}\right)$ is $0.240(7)$ for $L=24$. Nevertheless we observe that the pion data can be well fitted from $L=12$ with the "semi-empirical" formula

$M_{\pi}(L)=m_{\pi}+a_{1} \frac{3}{4(2 \pi)^{3 / 2}} \frac{m_{\pi}^{3 / 2}}{F_{\pi}^{2}} \frac{e^{-a_{2} m_{\pi} L}}{L^{3 / 2}}$

where $m_{\pi}, a_{1}$ and $a_{2}$ are free fit-parameters. $a_{1}$ and $a_{2}$ are chosen such that their deviation from 1 indicates "how far away" we are from the original formula (11). For the rho (which cannot decay on the employed lattices) and the nucleon we adopt, for various practical reasons, the generic ansatz

$M_{\rho, N}(L)=m_{\rho, N}+a_{1} \frac{e^{-a_{2} m_{\pi} L}}{L^{3 / 2}}$,

i.e. we effectively drop the term proportional to (3). Here, $m_{\pi}$ is fixed to the value obtained 
Table 1

GRAL production status as of $07 / 08 / 2003$

\begin{tabular}{|c|c|c|c|c|c|c|c|c|c|}
\hline$\beta$ & $\kappa$ & $L^{3} \times T$ & $N_{\text {conf }}$ & $\left\langle\frac{1}{3} \operatorname{Tr} \square\right\rangle$ & $r_{0}$ & $a_{r_{0}}^{-1}[\mathrm{GeV}]$ & $M_{\pi}(L) L$ & $M_{\pi} L$ & $L[\mathrm{fm}]$ \\
\hline \multirow[t]{3}{*}{5.32} & 0.1665 & $12^{3} \times 32$ & 4900 & $0.5395(4)$ & & & & & \\
\hline & & $14^{3} \times 32$ & 2300 & $0.5382(2)$ & & & & & \\
\hline & & $16^{3} \times 32$ & 3400 & $0.5382(1)$ & $3.84(3)$ & $1.51(2)$ & $4.3(2)$ & & \\
\hline \multirow[t]{8}{*}{5.6} & 0.1575 & $10^{3} \times 32$ & 6200 & $0.57324(7)$ & & & $4.9(2)$ & $2.76(3)$ & $0.850(4)$ \\
\hline & & $12^{3} \times 32$ & 7200 & $0.57280(5)$ & & & $4.41(8)$ & $3.32(4)$ & $1.020(5)$ \\
\hline & & $14^{3} \times 32$ & 6500 & $0.57262(4)$ & & & $4.23(7)$ & $3.87(4)$ & $1.190(6)$ \\
\hline & SESAM & $16^{3} \times 32$ & 6500 & $0.57254(3)$ & $5.96(8)$ & $2.35(3)$ & $4.49(6)$ & $4.43(5)$ & $1.360(7)$ \\
\hline & $\mathrm{T} \chi \mathrm{L}$ & $24^{3} \times 40$ & 5100 & $0.57248(1)$ & $5.89(3)$ & $2.32(1)$ & $6.64(7)$ & $6.64(7)$ & $2.04(1)$ \\
\hline & 0.1580 & $14^{3} \times 32$ & 4400 & $0.57367(4)$ & & & $4.0(2)$ & & \\
\hline & & $16^{3} \times 32$ & 3900 & $0.57345(5)$ & & & $3.7(2)$ & & \\
\hline & $\mathrm{T} \chi \mathrm{L}$ & $24^{3} \times 40$ & 4500 & $0.57337(2)$ & $6.23(6)$ & $2.45(2)$ & $4.78(7)$ & & \\
\hline
\end{tabular}

from the pion fit. All the fits are displayed in Fig. [1 where the lines are solid within the fit range and dotted outside. For comparison the dash-dotted curves show $1 / L^{3}$-fits of the form (4). The $\chi^{2} /$ dof-values for the exponential fits are $0.20,0.03$ and 1.33 for pion, rho and nucleon, respectively. In contrast, for the $1 / V$-fits $\chi^{2} /$ dof is 19,8 and 4 . The asymptotic mass $m_{\pi}\left(m_{\rho}, m_{N}\right)$ obtained from the fit deviates less than $1 \%$ from $M_{\pi}\left(M_{\rho}, M_{N}\right)$ at $L=24$ and lies thus well within the errors of $M_{\pi}\left(M_{\rho}, M_{N}\right)$.

\section{CONCLUSIONS AND OUTLOOK}

We clearly see an exponential behaviour in our data for the light hadrons, as opposed to the $1 / L^{3}$-dependence reported earlier by Fukugita et al.. However, the $1 / L^{3}$-fits are acceptable when we shift the fit-range from (12..24) towards smaller $L$, say (10..16). Moreover, we have to allow for extra free parameters in Lüscher's formula in order to achieve a good description of our data. These parameters are $O(1000)$ in the case of $a_{1}$ and about 2 in the case of $a_{2}$ (except for the nucleon where $a_{1}=O(100)$ and $\left.a_{2} \approx 1\right)$ and vary with the left fit boundary. This, together with the $F_{\pi} L$-values mentioned above, suggests that we are in an intermediate, sub-asymptotic regime were we merely see the onset of the chiral behaviour predicted by Lüscher's formula.
In a next step we will analyse our data at $\beta=5.6, \kappa=0.158$ and $\beta=5.32, \kappa=0.1665$ in order to check whether the observed volumedependence is reproduced there. We will then address the question if and to what precision an extrapolation to the infinite volume is possible.

\section{ACKNOWLEDGEMENTS}

The gauge configurations were produced on the cluster computer ALiCE at the University of Wuppertal and on the Quadrics/APE installation at DESY/NIC Zeuthen. Further numerical calculations were carried out on the Cray T3E at FZ/NIC Jülich. We thank the operating staffs in all these places for their support. BO thanks S. Dürr for useful discussions.

\section{REFERENCES}

1. M. Lüscher, DESY 83/116 Lecture given at Cargese Summer Inst., Cargese, France, Sep 1-15, 1983

2. M. Lüscher, Commun. Math. Phys. 104 (1986) 177.

3. M. Fukugita, H. Mino, M. Okawa, G. Parisi and A. Ukawa, Phys. Lett. B 294 (1992) 380.

4. M. Fukugita, N. Ishizuka, H. Mino, M. Okawa and A. Ukawa, Phys. Rev. D 47 (1993) 4739. 